Thorax (1973), 28, 637.

\title{
Behçet's syndrome with ulcerative oesophagitis: report of the first case
}

\author{
THOMASE. BRODIE and JOHN L. OCHSNER ${ }^{1}$ \\ Department of Surgery, Ochsner Clinic and Ochsner Foundation Hospital, \\ New Orleans, Louisiana 70121, U.S.A.
}

\begin{abstract}
Brodie, T. E., and Ochsner, J. L. (1973). Thorax, 28, 637-640. Behçet's syndrome with ulcerative oesophagitis: report of the first case. A patient is presented who has the typical manifestations of Behçet's syndrome-oral, perianal, and skin ulcerations, arthritis, and thrombophlebitis. His presenting symptom, consisting of severe dysphagia caused by oesophageal ulceration, is unreported in connection with this disease. The ulceration became a perforation requiring surgical treatment. His malnourished state and long-term steroid therapy made him a poor surgical risk and preoperative and postoperative nutritional and respiratory support were necessary. Prophylaxis against thromboembolic phenomena is indicated postoperatively in these patients.
\end{abstract}

Behçet (1937) described a chronic disease which manifested itself as an acute, relapsing, episodic entity and consisted of three symptoms-ulceration of the mouth, ulceration of the genitalia, and severe iritis. Two of the original three symptoms are now considered sufficient to establish a diagnosis. Behçet's syndrome appears to be an autoimmune disease and numerous additional manifestations have been described. It has occurred in association with arthritis (Oshima et al., 1963), colitis (Bøe, Dalgaard, and Scott, 1958 ; Ramsay, 1967), skin lesions, thrombophlebitis, and encephalitides (O'Duffy, Carney, and Deodhar, 1971). This paper reports a previously undescribed manifestationdysphagia with severe oesophageal ulceration.

\section{CASE REPORT}

A 71-year-old white man was admitted to Ochsner Foundation Hospital in October 1970 for severe acute dysphagia of one week's duration. He had been in hospital several times previously for treatment of ulceration of the mouth, thrombophlebitis, and rheumatoid arthritis. Steroid therapy had been started in November 1967.

An oesophagogram showed dilatation of the distal oesophagus with oesophageal ulceration. A biopsy showed severe acute oesophagitis. Biopsy examination of a perianal and oral ulcer showed nonspecific inflammatory reaction with ulceration and an infiltrate of lymphocytes and some plasma cells. A raised papule on the left knee, although showing no ulceration,

IReprint requests to Dr. Ochsner, c/o Ochsner Clinic, 1514 Jefferson Highway, New Orleans, Louisiana 70121, U.S.A. showed a marked perivascular infiltrate of lymphocytes. It was concluded that the symptom complex was consistent with Behçet's syndrome. He responded to medical therapy and was discharged without symptoms and with a radiologically normal oesophagus.

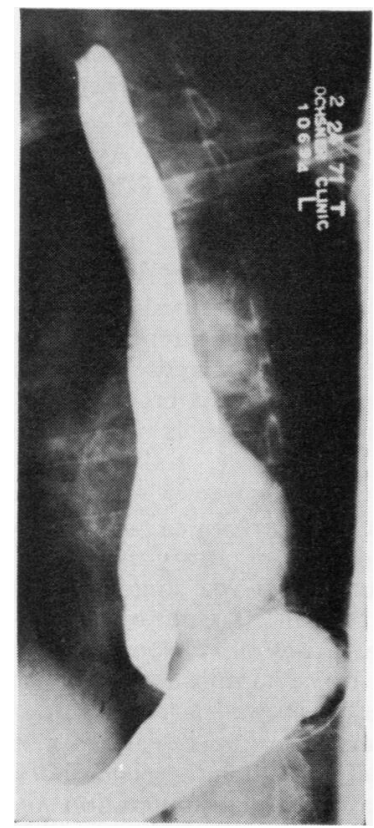

FIG. 1. Oesophagogram showing ulceration of the distal one-third of the oesophagus with mediastinal abscess. 


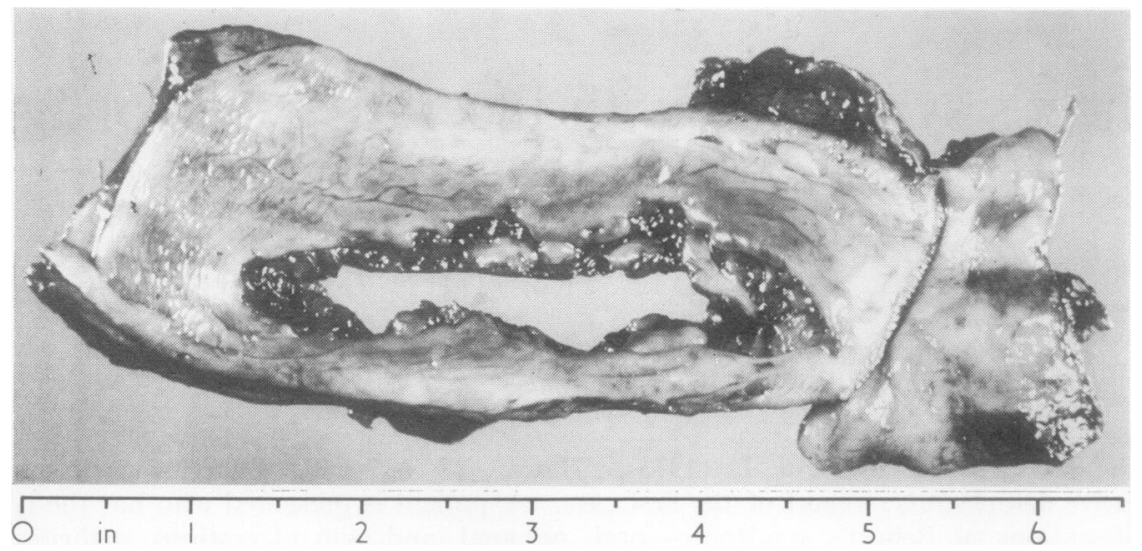

FIG. 2. Surgical specimen showing $7 \mathrm{~cm}(3.5 \mathrm{in})$ linear ulcer in the posterior oesophageal wall.

In October 1971 he was readmitted following an acute recurrence of the dysphagia. An oesophagogram showed dilatation of the distal oesophagus with areas of ulceration. The patient had gradually modified his diet so that he was then taking only soft, puréed food and was suffering from considerable regurgitation with some vomiting. Again, he was managed medically.

Three months later he was readmitted. He was severely debilitated and had not eaten solid food for a year and a half. He had not been able to walk without assistance for six months. Significant physical findings included a mild to moderate ankle oedema, severe orthostatic hypotension, and general debility.

Laboratory studies revealed a severe neutropenia of $2.4 \times 10^{3}$ white blood cells, a markedly contracted red cell volume, but normal haemoglobin and haematocrit, a significantly contracted blood volume, and a significant hypoproteinaemia of $5.8 \mathrm{~g}$ with a serum albumin of $2 \cdot 2 \mathrm{~g}$.

Radiological evaluation of the lower end of the oesophagus showed that a narrowing of the oesophagogastric junction had remained relatively unchanged during the preceding three months, whereas the lower part of the thoracic oesophagus was widened and gave the appearance of being external to the oesophagus. This finding was interpreted as being a possible abscess cavity (Fig. 1); however, malignancy could not be excluded and oesophagoscopy was recommended.

Oesophagoscopy performed on 6 December 1971 revealed marked inflammatory exudate with considerable granulation tissue and some inflammatory polyp formation at $30 \mathrm{~cm}$. Tissue from the ulcer crater showed severe, acute on chronic inflammation with necrosis but a tissue sample taken immediately proximal to this lesion showed a normal oesophagus.

Surgical intervention was planned but, because of the patient's debilitated state and blood volume deficiency, preoperative hyperalimentation and transfusion were necessary. Oesophagectomy with oesophagogastrostomy was performed on 16 December 1971. A linear ulcer, $3 \times 7 \mathrm{~cm}$, had penetrated all layers of the distal posterior oesophageal wall (Fig. 2). The base of the ulcer was formed of granular tissue in the posterior mediastinum, in effect a large mediastinal abscess. Twelve centimetres of the distal oesophagus and the oesophagogastric junction were removed.

The pathology report indicated that the ulcer penetrated the mucosa and submucosa into the muscular coat. The base of the ulcer contained granulation tissue with proliferating fibroblasts, lymphocytes, polymorphonuclear leucocytes, and occasional plasma cells. The histological diagnosis was chronic nonspecific ulcer of the oesophagus.

The patient's postoperative course was complicated by marked hypoventilation, bilateral pneumonia, and post-traumatic pulmonary insufficiency. These complications were vigorously treated and the patient responded slowly but consistently. He was discharged one month postoperatively, taking food orally with no dysphagia or regurgitation, walking with a walking frame, and without any other manifestation of Behçet's syndrome but maintained on his preoperative steroid regime.

The patient was readmitted on 2 February 1972 for management of an acute exacerbation of the mouth ulceration. He had had no recurrence of the dysphagia or regurgitation. His hospital course was benign until $N$ 27 February when he died suddenly of pulmonary or emboli involving the main and small branches of $N$ both pulmonary arteries.

\section{DISCUSSION}

This patient presents an interesting problem from $\mathbb{D}$ both a diagnostic and a therapeutic point of view. From his history of a chronic relapsing symptom complex consisting of oral and perianal ulcera- $\frac{O}{\mathbb{D}}$ tions, arthritis, thrombophlebitis, skin lesions, and $\frac{\odot}{\Phi}$ oesophagitis, Behçet's syndrome could be con- $\mathbb{\AA}$ templated as a diagnosis. When Behçet (1937) श् originally described this disease, he described an 8 
associated ocular inflammation but this symptom at no time could be documented in this patient.

No description of oesophageal manifestations of Behçet's syndrome could be found in the literature, although Arma, Habibulla, Price, and Collis (1971) did describe the appearance of dysphagia with the typical symptom complex. However, in their report there was no associated ulceration and there was an aganglionic segment of the oesophagus. In our patient there was no such aganglionic segment and the oesophageal lesion was histologically consistent with Behçet's syndrome.

The oesophageal ulceration did not appear to be due to reflux of gastric juices. The operative specimen showed none of the fibrosis and constriction usually associated with peptic oesophagitis and there was no evidence of a hiatal hernia either at operation or in preoperative radiological studies. Microscopically, the oesophagus was entirely normal up to the margin of the ulcer. Histological sections from the ulcer wall showed changes consistent with a nonspecific ulcerative inflammatory process and the infiltrate, which contained both plasma cells and chronic inflammatory cells, was consistent with the changes found in other ulcers elsewhere in the body (Fig. 3). Therefore the ulceration in this patient's oesophagus probably was a manifestation of Behçet's syndrome.

The patient's malnourished state and long-term steroid therapy made him a poor surgical risk and a significant management problem. However, intravenous hyperalimentation, using the method described by Dudrick, Long, Steiger, and Rhoads (1970), was successful in restoring the patient sufficiently for the operation. The preoperative steroid preparation of the patient was parenteral hydrocortisone, which was continued postoperatively. He was discharged on the same steroid dosage as before the operation. The long-term steroid therapy, in our opinion, did play a significant part in the progression of the disease of the oesophagus. The ulcer probably did not heal initially because of the immunosuppressive and anti-inflammatory effect of the steroid. In addition, it suppressed the symptoms, probably causing delay in treatment.

The operative procedure was performed through separate abdominal and right thoracic incisions (Ong, 1971). The perforation in the oesophageal wall, indicating a contaminated field, necessitated some modification of technique. After vigorous debridement of the granular mass, which repre-

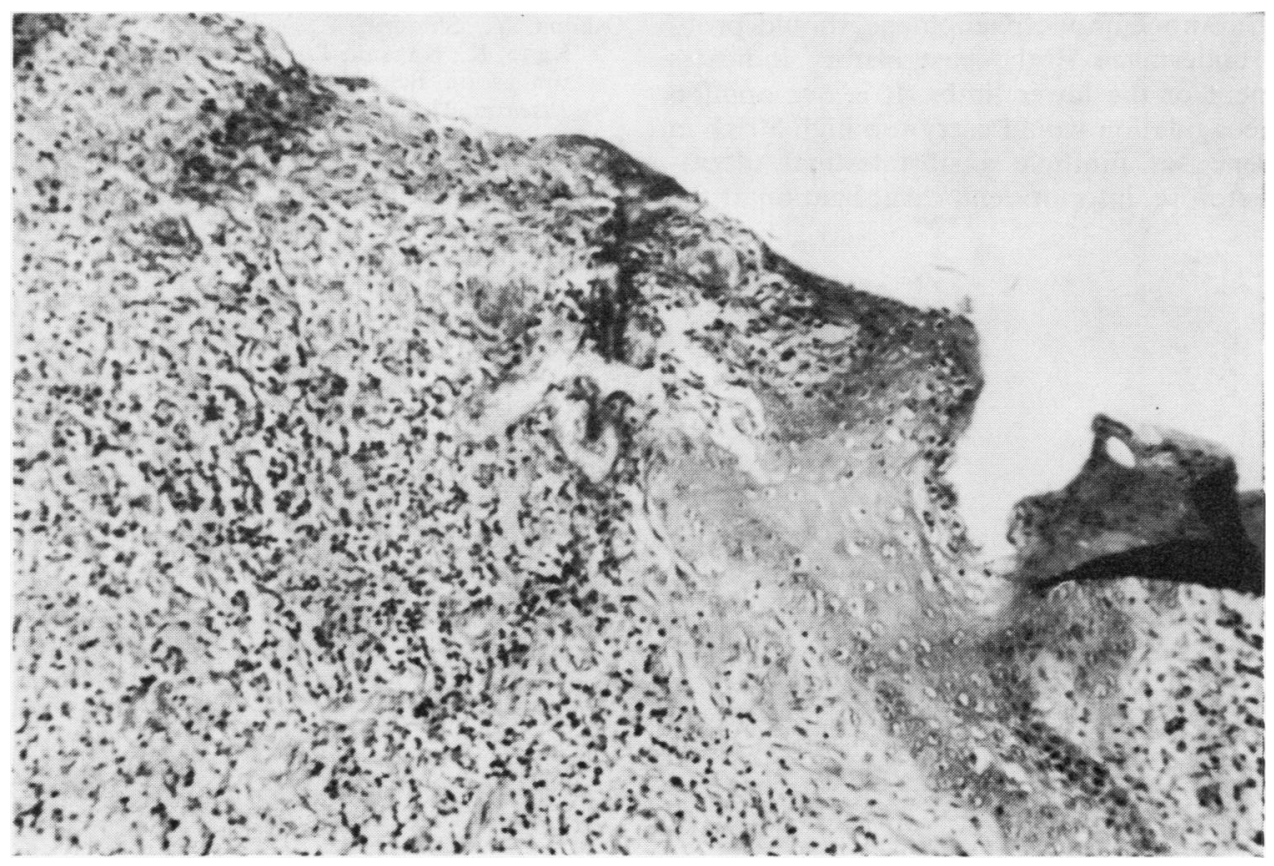

FIG. 3. Photomicrograph showing ulcer penetrating the mucosa and submucosa into the muscular coat. The base of the ulcer contains granulation tissue with proliferating fibroblasts, lymphocytes, polymorphonuclear leucocytes, and occasional plasma cells. Haematoxylin and eosin $\times 77$. 
sented the wall of the mediastinal abscess, the entire area was copiously irrigated with an antibiotic solution. The oesophagogastric anastomosis was performed at the level of the tracheal bifurcation. The possibility of using a colonic transplant was entertained; however, colonic manifestations of Behçet's syndrome are described in the literature (Ramsay, 1967) and this patient had complained of severe diarrhoea on a number of occasions. He also had had an abnormal barium enema examination which is consistent with the colitis without ulceration described by Ramsay (1967). In view of these factors it was decided that the stomach should be used as a replacement for the oesophagus. The mediastinum was allowed to remain open to the right pleural cavity and was drained via this space by thoracotomy tubes placed to suction under water.

The patient's subsequent death is significant since thrombophlebitic phenomena are well described in Behçet's syndrome and subsequent pulmonary emboli are always a hazard. It is difficult to determine in this case whether the emboli described at necropsy originated in the lower limbs or more proximally, as obstruction of both superior and inferior venae cavae has recently been described (Kansu et al., 1972). Prophylaxis against thromboembolic phenomena should probably be undertaken if the past history indicates involvement of the lower limbs. It is our opinion that anticoagulation would carry too high a risk in the presence of multiple gastrointestinal ulcerations ; therefore, inferior vena caval ligation at the time of surgery would be the treatment of choice. As the embolism in our patient occurred some two and a half months after his operation, and while he was ambulatory, it probably was a complication of the disease rather than of the operation.

\section{REFERENCES}

Arma, S., Habibulla, K. S., Price, J. J., and Collis, J. L. (1971). Dysphagia in Behçet's syndrome. Thorax, 26, 155.

Behçet, H. (1937). Uber rezidivierende, Aphthöse, durch ein Virus verursachte Geschwüre am Mund, am Auge und an den Genitalien. Dermatologische Wochenschrift, $105,1152$.

Bøe, J., Dalgaard, J. B., and Scott, D. (1958). Mucocutaneousocular syndrome with intestinal involvement. American Journal of Medicine, 25, 857.

Dudrick, S. J., Long, J. M., Steiger, E., and Rhoads, J. E. (1970). Intravenous hyperalimentation. Medical Clinics of North America, 54, 577.

Kansu, E., Özer, F. L., Akalin, E., Güler, Y., Zileli, T., Tanman, E., Kaplaman, E., and Müftüoglu, E. (1972). Behçet's syndrome with obstruction of the venae cavae: A report of seven cases. Quarterly Journal of Medicine, 41, 151.

O'Duffy, J. D., Carney, J. A., and Deodhar, S. (1971). Behçet's disease. Report of ten cases, three with new manifestations. Annals of Internal Medicine, 75, 561.

Ong, G. B. (1971). Resection and reconstruction of the esophagus. Current Problems in Surgery, 3, 56.

Oshima, Y., Shimizu, T., Yokohari, R., Matsumoto, T. Kano, K., Kagami, T., and Nagaya, H. (1963). Clinical studies on Behçet's syndrome. Annals of Rheumatic Diseases, 22, 36.

Ramsay, C. A. (1967). Behçet's syndrome with large bowel involvement. Proceedings of the Royal Society of Medicine, 60,185 . 